\title{
Examination of Interfacial Reactions of a NiAl-Hf-hBN System on a Sapphire Fibre by a Combination of EPMA and FIB Specimen Preparation
}

\author{
S. Richter*, S. Kyrsta**, D. Hajas**, J. Schneider **and J. Mayer* \\ *Central Facility for Electron Microscopy (GFE), RWTH-Aachen, D-52056 Aachen, Germany \\ **Institute of Materials Chemistry(MCh), RWTH-Aachen, D-52056 Aachen, Germany
}

Fibre reinforced NiAl offers new possibilities for the development of high strength structural materials of low density applicable in gas turbines at high operating temperatures. The properties of composite materials are strongly influenced by the strength of the fibre-coating interaction. In addition, if fibre and coating differ in their thermal expansion coefficients interface layers are demanded due to high temperature fluctuations. Therefore, two layers consisting of BN and Hf were embedded between a sapphire fibre and $\mathrm{NiAl}$ and heated at $1350 \propto \mathrm{C}$ to find a compromise between adhesion and ductility. The control and characterization of the reaction zone is essential for the development of these new materials under investigation in our laboratory. Especially, the characterization of the fibre-coating interface is a challenge. The different hardness of fibre and coating makes it nearly impossible to use a conventionally cross-section preparation. Further, the small dimension of the reaction zone requires the use of analytical techniques providing high lateral resolution. In order to accomplish these requirements, a new developed technique based on FIB (Focused Ion Beam)-preparation and EPMA (Electron Probe Microanalysis) was combined with XRD (X-ray diffraction). EPMA is a well-established method in quantifying mass coverages of elements in thin layers with high accuracy [1]. One step further, the method can be expanded to the analysis of multilayered structures by removing the material in a controlled manner [2]. Thus, the whole multilayered structure can be opened and finally analysed by EPMA. The focused ion beam technique provides a controlled removal of the material on the Nanometerscale. This technique was applied in our work. The aim of this paper is to present this new combination of methods for the characterization of a complex structure with regard to geometry and chemistry.

The crystallographic structure of films and formed phases was analysed by means of X-ray microdiffraction in a Bruker D8 Discover diffractometer with GADDS (general area detector diffraction system). Phases of $\mathrm{HfB}_{2}, \mathrm{HfN}, \mathrm{AlN}, \mathrm{NiAl}$ and $\alpha-\mathrm{Al}_{2} \mathrm{O}_{3}$ were identified. The samples were fractured by bending the composite specimen along the fibre axis. The fracture exposes the fibre coating interface in such a way that the BN-layer was on the surface.

The FEI Strata 205 Focused Ion Beam was used for the FIB specimen preparation. The ion beam was focused on the cleavage plane with an angle of about $2 \infty$ relatively to the surface of NiAL-HfBN-system. A bevel was milled by scanning the beam over an area of about $2 * 20 \mu \mathrm{m} \leq$ (Fig. 1).The uncovered NiAL-Hf-BN-system can be seen in Fig. 2 on top view by secondary electron imaging. EPMA measurements were performed by a Camebax SX 50 along the bevel in two modes: (1) the elemental mapping and (2) a quantitative linescan. The X-ray mappings of the elements $\mathrm{N}, \mathrm{Al}, \mathrm{Ni}$, $\mathrm{O}$, Hf and $\mathrm{B}$ acquired across an area of the milled bevel are shown in Fig. 3. The orientation of the NiAl-Hf-BN system is NiAl at the top,Hf in the middle and $\mathrm{BN}$ at the bottom. In contrast to the analysis of a cross section, where the interfaces of a layered system is aligned with the electron beam direction, the analysis along a bevel needs a special interpretation due to the relatively large information depth of the X-rays. 
For the quantitative information characteristique X-ray intensities were measured by scanning the electron beam along a line with a step width of $1 \mu \mathrm{m}$ at a beam energy of $10 \mathrm{keV}$ (Fig. 4a). The kratios, i.e. the calibrated net $\mathrm{X}$-ray intensities from the sample to those of bulk standards, are plotted versus distance starting from the uncovered NiAl-coating. Furthermore with knowledge of all the above mentioned results the reaction zone was reconstructed by a simplified thin film algorithm assuming a laterally homogeneous multilayered structure (see Tab.1). To prove the validity of the reconstruction, intensity profiles (Fig. 4b) were calculated using a Monte-Carlo-SimulationSoftware[2]. The good agreement encourages a further development of the new technique.

\section{References}

[1] N. Ammann et al.(1992), Mikrochim.Acta 12, 213-219.

[2] S. Richter et al.(1998), Mikrochim. Acta 15, 125-131.

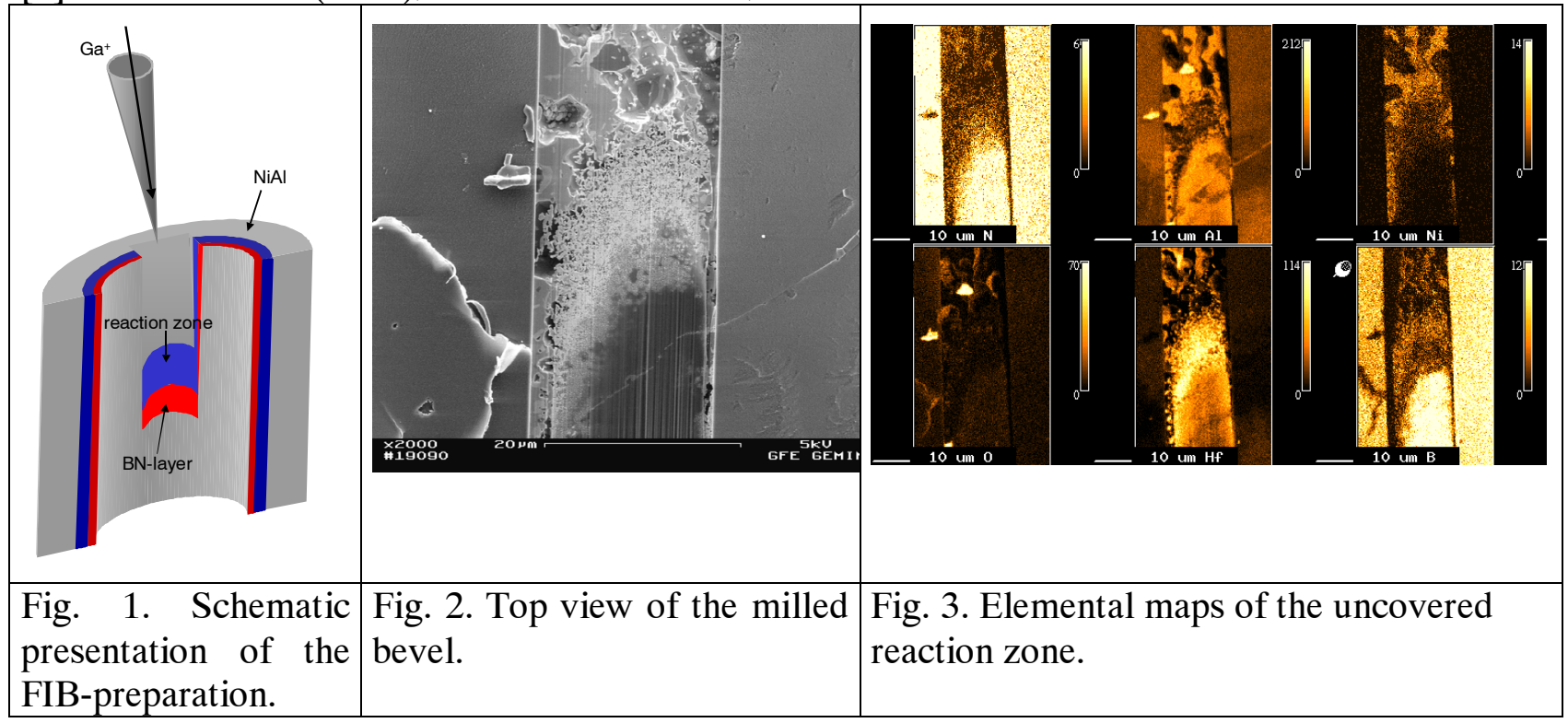

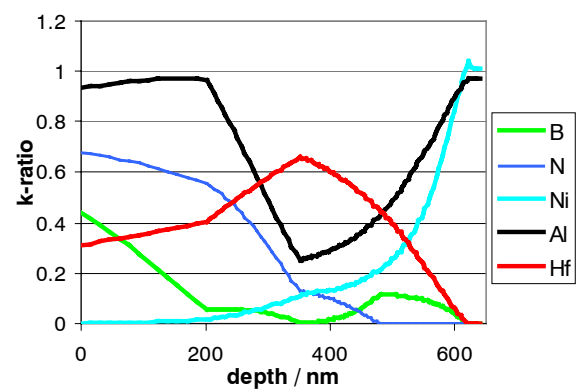

a)

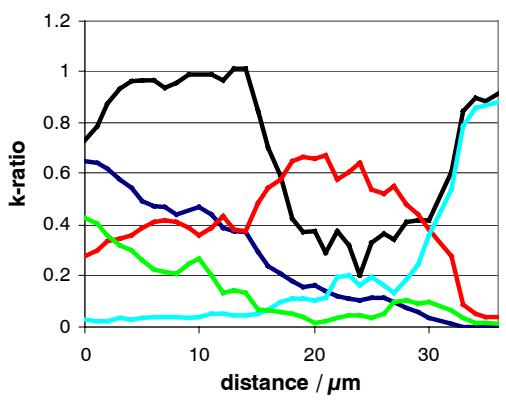

b)

Fig. 4.: Bevel line scan measurements of calibrated X-ray intensities (b) compared with the ones calculated by Monte-Carlo-Simulation (a).

Table1.: Reconstructed chemical composition and thickness of each layer.

\begin{tabular}{|c|c|l|l|l|}
\hline BN-layer & \multicolumn{3}{|c|}{ Reaction zone } & NiAl-substrate \\
\hline $\begin{array}{c}\text { B58N42 } \\
/ \text { at } \%\end{array}$ & $\begin{array}{c}{[\text { AlN]67[BN]33 }} \\
/ \text { at } \%\end{array}$ & $\begin{array}{l}{[\mathrm{HfN}] 64[\mathrm{AlN}] 8} \\
\text { Ni13Al15 } / \mathrm{at} \%\end{array}$ & $\begin{array}{l}{[\mathrm{HfB} 2] 60} \\
\text { Ni20A120 /at } \%\end{array}$ & $\begin{array}{c}\text { Ni51Al49 } \\
/ \text { at } \%\end{array}$ \\
\hline & $114 \mathrm{~nm} ; 3.9 \mathrm{~g} / \mathrm{cm} \geq$ & $130 \mathrm{~nm} ; 7.3 \mathrm{~g} / \mathrm{cm} \geq$ & $140 \mathrm{~nm} ; 7.8 \mathrm{~g} / \mathrm{cm} \geq$ & \\
\hline
\end{tabular}

